\title{
Bodies recovered from water: a personal approach and consideration of difficulties
}

W Lawler

\section{Introduction}

For the pathologist providing a routine necropsy service to the local coroner, examination of bodies recovered from water can generate the most difficult of interpretational problems, and this is probably the prime context where appropriate historical and circumstantial evidence is vital to interpretation and overall conclusions, ${ }^{12}$ although such collateral evidence should always be available before any coroner's necropsy is undertaken. ${ }^{3}$

It must be appreciated, at the outset, that not all persons whose bodies are recovered from water will have died from its inhalation, although they may show features reflecting immersion in water. Such bodies should therefore be particularly carefully examined, both externally and internally, to catalogue (and subsequently to explain satisfactorily) all injuries present, to determine whether death indeed followed immersion in the water, and to see whether any natural disease, such as ischaemic heart disease, cerebrovascular disease, and hypertension, may have contributed to, precipitated, or even caused death. It is also important to determine whether the deceased was under the influence of alcohol or other drugs at the time of death (although interpretation of laboratory results should be influenced by the knowledge that, as discussed below, classic fresh water drowning may increase the blood volume by as much as $30-35 \%)$. Finally, the pathologist has a vital role in determining, from all pathological and circumstantial evidence available, whether the overall findings are consistent with, or even point directly towards accident, suicide, or homicide.

Unfortunately many bodies recovered from water will have been there for several days, and decomposition may have obscured or destroyed features of drowning; nevertheless, careful examination may elicit sufficient positive or negative findings to allow reasonable conclusions to be drawn.

For the pathologist to interpret accurately the necropsy findings, it is necessary briefly to consider the mechanisms of death after submersion in water and to appreciate the results of immersion in water, including artefactual injuries.

\section{Mechanisms of death after submersion in water}

These are well documented in several standard textbooks of forensic medicine and pathology. ${ }^{4-8}$
It has been reported that about $85-95 \%$ of those dying from water inhalation present features of drowning, ${ }^{59}$ although in most, not all the typical features are seen ${ }^{9}$; the remainder die from vagal inhibition (sometimes, inaccurately, known as "dry drowning", and once designated "hydrocution"), or the post immersion syndrome; perhaps, rarely, laryngeal spasm may be important.

At this stage, it is worth remembering that hypothermia can supervene very quickly in individuals swimming or trying to remain afloat in cold water, and that it may be an important factor contributing to their death $^{1011}$; indeed, hypothermia may be the main cause of death after shipwreck in the open sea. ${ }^{1112}$

\section{DROWNING}

Mechanisms for death from drowning are multiple, complex, and, in part, still incompletely understood. Although drowning is much more than simple asphyxia following mechanical airway obstruction by water, this process probably does at least contribute. Major factors, however, seem to be osmotic and perhaps also hydrostatic effects of the inhaled fluid once it reaches alveolar spaces and gains access to semipermeable alveolar membranes; here, water and electrolyte exchanges take place, the nature of which is influenced by the tonicity of the inhaled fluidfresh or salt water.

Fresh water This is hypotonic relative to plasma. Therefore, when present in alveoli, it is rapidly absorbed into the pulmonary circulation; this causes pronounced haemodilution (the blood volume may be increased by up to $30-35 \%$ ) which, in turn, soon produces local haemolysis. Although haemodilution will lead to hyponatraemia, circulatory overload, and, ultimately, high output cardiac failure, haemolysis is probably more important, as it causes hyperkalaemia and consequent cardiac arrhythmias, particularly with concomitant generalised hypoxia. These changes can develop very rapidly-over a few minutes, supporting the view that drowning in fresh water tends to occur more quickly than in sea water. $^{56}$

Salt water is hypertonic relative to plasma. Therefore, when present in alveoli, it attracts water into the airways from the pulmonary circulation, causing local haemoconcentration and severe pulmonary oedema. Haemoconcentration increases blood viscosity and pro- 
duces acute hypernatraemia, while severe pulmonary oedema causes clinically important hypoxia/hypercapnoea; all these factors adversely affect the heart, with bradycardia and, ultimately, asystole.

"VAGAL INHIBITION" ("REFLEX CARDIAC ARREST") This is a well recognised and accepted mechanism, particularly since Simpson's widely quoted review. ${ }^{13}$ Vagus nerve branches may be stimulated in several ways, with a direct and perhaps almost instantaneously fatal cardiac inhibition. Following submersion, it may be initiated by the sudden and unexpected entry of water into the larynx, nose, or nasopharynx ${ }^{414-16}$; concurrent emotional states may act as a contributing sensitising factor. ${ }^{16}$ Vagal inhibition seems to be more common when the submersion is total and unanticipated, when the victim is under the influence of alcohol and/or other drugs, when the water is cold, and when the individual enters it feet first. ${ }^{15}$

LARYNGEAL SPASM

This probably occurs, at least to some extent, in most individuals following submersion, as it presumably represents a normal reflex to fluid entering the larynx..$^{61517}$ In most, however, it seems to be transient, and a true asphyxial death from laryngeal spasm, if it occurs at all, is probably extremely rare. Gardner reports having seen only one fatal case-in a boy aged 8 who sank into water immediately after jumping in, and whose body showed asphyxial changes and no features of drowning. ${ }^{15}$ This mechanism is discussed by Polson, Gee, and Knight, ${ }^{6}$ who quote Gardner's case but do not offer any of their own; they do, however, state that laryngeal spasm is "a rare mode of death from submersion." Several reviews ${ }^{12} 16$ do not mention it at all; some, illogically, link it with vagal inhibition as a mechanism for almost instantaneous death, and do not refer to asphyxial features. ${ }^{457} \mathrm{My}$ views, and, I believe, those of many colleagues involved in forensic pathology, are well summarised by Donald, ${ }^{17}$ who says "previous literature would suggest that a number of human beings are drowned with dry lungs owing to glottic spasm, but little convincing evidence has been produced". Recently, Knight has stated "another mechanism that is often postulated as a cause of nondrowning immersion death is 'laryngeal spasm', leading to a hypoxic death from closure of the airway. ${ }^{8}$ The evidence for such a condition is tenuous, as such closure would have to operate for a considerable time for hypoxia to kill, all the time keeping the larynx closed to prevent entry of water."

POST IMMERSION SYNDROME (SECONDARY DROWNING)

Occasionally, individuals survive the immersion and are recovered alive from the water, only to die later from delayed effects or other complications. Such deaths are usually pulmonary, reflecting surfactant loss following fluid inhalation; some represent prolonged, profound hypoxia. ${ }^{1819}$

\section{Findings and interpretations in deaths} after submersion in water

It is important to distinguish changes directly attributable to death following submersion (discussed here) from those which purely reflect immersion (discussed later). The changes described here, which are well documented in standard textbooks ${ }^{4}$ and review articles, ${ }^{1820}$ are those encountered in fresh bodies-that is, those removed from the water before decomposition becomes established; once a lengthy delay has occurred, positive diagnosis may be difficult.

\section{DROWNING}

Externally, although a range of changes may be identified, there may be nothing specific to drowning. Sometimes, however, firm, tenacious foam is present at the mouth or nostrils. Typically, it is white or blood tinged, and reappears after wiping away. It is thought to represent an admixture of air, fluid, mucus and surfactant, and therefore an ante mortem phenomenon.

Internally, the foam, even if not apparent externally, is often found in major airways or secondary bronchi and bronchioles. The airways may also contain water and such extrinsic materials as silt, weeds, or sand. Similar substances (particularly water) may be swallowed and thus identified within the stomach. Pulmonary changes vary according to the drowning fluid, although they are often not as distinct as suggested by differences in causative mechanisms and as implied in some textbooks.

\section{Fresh water}

Typically, the lungs are almost twice their normal weight, and present an appearance sometimes still designated "emphysema aquosum"-they are bulky and overdistended (such that they may well overlap the pericardial sac and meet in the midline), with a very characteristic doughy texture which causes them to pit on digital pressure and sometimes to show prominent rib markings. Classic petechial haemorrhages are uncommon, but larger subpleural and intrapulmonary haemorrhages may be identified. Section releases frothy, often blood tinged fluid. Elsewhere, haemodilution causes the blood to appear rather "watery"; haemolysis may produce intimal staining of major vessels.

\section{Salt water}

Typically, the lungs are slightly, but not always significantly, heavier than in fresh water drowning, ${ }^{21}$ and although overdistended, classic emphysema aquosum is less pronounced; on section, greater quantities of frothy fluid tend to be released. Pleural effusions may also be present.

\section{VAGAL INHIBITION}

This is really a diagnosis of exclusion based not only on negative pathological and toxicological findings, but also on appropriate circumstantial evidence; necropsy shows no foam in the airways, no emphysema aquosum, no 
petechial haemorrhages and no clinically relevant natural disease. ${ }^{8 \times 1315}$

LAYRNGEAL SPASM

Here, presuming the existence of this entity, the features are those of classic mechanical asphyxia, including cyanosis, congestion, and widespread petechial haemorrhages; there is little or no water in the airways (although some may be present in the stomach), no or minimal airway froth, and no emphysema aquosum. ${ }^{6}{ }^{15}$ Such findings indicate the possibility of an asphyxial death before entry into the water which must be actively and seriously considered, as this mechanism for death following submersion is extremely rare, if it exists at all.

POST IMMERSION SYNDROME (SECONDARY

DROWNING)

With short term survival, lungs develop haemorrhagic, desquamative bronchopneumonia, with intra-alveolar hyaline membranes; later, abscesses may develop, and granulomatous reactions to inhaled foreign particles may be identified. $^{1819}$ Simultaneously, there may be hypoxic damage elsewhere, particularly in cerebrum, brain stem, and renal tubules.

\section{"Confirmatory" tests for drowning}

Two are often quoted as providing evidence for drowning. In practice, both are difficult to perform and to interpret, with many false positive and false negative results. ${ }^{2}$

\section{DIATOMS}

This subject has generated much debate and controversy, with strong arguments in favour of and against diatom identification as a helpful diagnostic test; review articles are available, ${ }^{22-2526}$ and the subject has been discussed in standard textbooks. ${ }^{158}$ Diatoms (Bacillariophyceae) are unicellular algae with hard silicaceous exoskeletons resistant to decomposition, heat, and acids strong enough to destroy soft tissues. Over 10000 species and types exist, about half in fresh water and half in brackish or sea water; unfortunately, they are not found in substantial numbers all year round, the peaks being spring and autumn. In theory, drowning should allow diatoms to enter not only the lungs, but also, via the circulation, other organs. Therefore, in the drowned, diatoms should be extractable, after tissue digestion in strong acids, from such remote sites as bone marrow, liver, brain and kidneys. Unfortunately, two main problems exist: first, there may be insufficient or even no diatoms in the drowning fluid-from seasonal variations as noted above or following pollution by effluent-second, when identified, they may represent "contamination", such as during necropsy, from tap water, from reagents, from food via the deceased's gastrointestinal tract or even from the atmosphere. At best, despite strict, proper techniques and appropriate controls ${ }^{23}$ the diatom test can only provide supportive evidence of drowning. ${ }^{25}$ Such reservations probably apply even when experienced diatomologists undertake taxonomic analyses and comparisons of test and control samples. $^{2627}$

ELECTROLYTES

The haemodilution of fresh water drowning or the haemoconcentration of salt water drowning may be reflected in different electrolyte (particularly chloride ion) concentrations and plasma osmolarity or specific gravity between the different sides of the heart, ${ }^{28}{ }^{29}$ although most workers find these tests unreliable and quite unhelpful ${ }^{28121630}$; furthermore, as both are invalidated by decomposition, they can only be of any possible value in bodies recovered soon after death. ${ }^{4022}$

\section{Effects of immersion in water}

These, reviewed in standard texts, ${ }^{459}$ are obviously influenced by duration and water temperature, but other factors, such as whether the water is still or flowing, fresh or salt, clean or polluted, are also relevant.

Immersion modifies most changes after death. Body cooling will relate directly to the water temperature. In the United Kingdom cooling in water is roughly twice that in air, and is accelerated in flowing rivers and streams. Onset and duration of rigor mortis are also affected by water temperature: in cold water onset is delayed and duration prolonged. Drowning is a well recognised context in which cadaveric spasm (instantaneous rigor) may be encountered ("the drowning man clutching at straws"). As most submerged bodies float prone, with arms and legs hanging downwards, hypostasis (lividity) is usually maximal on face, neck, upper anterior chest, forearms, hands, lower legs and feet. In Caucasians it may be appreciably pink, perhaps because immersion facilitates oxygenation through the wet skin after death, ${ }^{7912}$ or perhaps merely the result of cold. ${ }^{8}$ With fast flowing water, the constant movement may impair, if not inhibit completely, development of hypostasis.

Decomposition (putrefaction) is also influenced by water temperature. In the United Kingdom time intervals associated with the various standard changes are about twice as long as those in air, but may be prolonged further in flowing water and reduced in heavy pollution. In tropical waters decomposition may be established by 24 hours, whereas none may be apparent after several weeks in water constantly below $40^{\circ} \mathrm{F}\left(5^{\circ} \mathrm{C}\right)$. With advancing decomposition, gas formation increases buoyancy until ultimately (in the United Kingdom after about three to 14 days, depending on the season), ${ }^{9}$ and providing it is free to do so, the body will float, often, because of intestinal putrefactive gases, belly upwards. ${ }^{49}$ Interestingly, once a submerged body is exposed to air after recovery, decomposition often proceeds very rapidly, and this may well continue despite apparently adequate refrigeration. ${ }^{46912}$ With prolonged immersion, adipocere will form.

Maceration, the skin change which characterises immersion, is due to water absorp- 
tion. ${ }^{689}$ It first appears on finger tips, and then involves the palm followed by the back of the hand; similar changes soon affect feet and skin elsewhere. The skin becomes whitened, sodden, thickened and wrinkled (an appearance sometimes designated "washerwoman's skin"). With time, the epidermis becomes loose and peels; finally, nails and hair become detached. Maceration is accelerated in warm water (where it may appear within minutes), but, in general, it takes about eight to 24 hours for early changes to become apparent outdoors in temperate climes. By about seven to 10 days, epidermal separation may have started, and by about three to four weeks, the skin and nails may be sufficiently loose to allow removal like a glove. Clothing, including footwear, delays maceration, perhaps by up to $50 \%$.

Following the above observations and comments, it is obvious that considerable variation exists between the different changes; consequently, it is extremely difficult to estimate the duration of immersion, and great care needs to be exercised when trying to draw reasonable conclusions. ${ }^{4}$

\section{Artefactual injuries during immersion in water}

These are common, and may provide interpretational difficulties. ${ }^{14-9}$ As most submerged bodies float prone, with arms and legs hanging downwards, contact with the rough bed of the stream, river, lake or sea will produce abrasions maximal over forehead, backs of hands, knees and toes. Tides or currents may crush the body against fixed objects, such as rocks, bridges, quays, weirs, wharfs and piers or ships; propellers may also inflict considerable damage.

Exposed skin may be bitten or chewed by fish, shellfish, and other marine life including aquatic mammals, and some creatures are able to gain access to skin below loose clothing. Occasionally, such large marine animals as sharks cause extensive lesions.

Although not always artefactual, serious injuries may be sustained either before the water was reached (on projecting rocks, pier pilings, bridge supports and quaysides) or while entering the water, especially after falling or jumping from a considerable height. The force generated by the latter may be sufficient to rupture internal organs.

\section{A personal approach to pathological conclusions}

As stated earlier, the pathological examination of a body recovered from water and the drawing of reasonable and justifiable inferences from the findings can be difficult. ${ }^{2}$ Each case has to be considered on merit, but it is essential that all circumstances-how and where the body was found, whether there were any local factors preventing the deceased extricating himself from the area involved, the mental and physical state of the deceased when last seen alive, the deceased's background medical history and even, perhaps, the deceased's swimming ability - are known by the pathologist before starting the necropsy. Indeed, most experienced pathologists would agree that this is one of the few areas where collateral evidence can be vital when trying to reach the most appropriate conclusions.

I believe that four groups of questions must be addressed and answered by the pathologist:

(1) What injuries are present on and within the body? How can each be explained satisfactorily? Consideration needs to be given to the possibility of artefactual injuries as discussed above. The likelihood that some, most, or even all the injuries identified were deliberately inflicted by an assailant must always be borne in mind, and may need appropriate investigation and active exclusion.

(2) What natural diseases are present? May they have produced sudden collapse and thus either caused death or precipitated drowning? Here, not only obvious structural abnormalities, such as ischaemic heart disease, cerebrovascular disease, and hypertension, but also functional disorders, the existence of which is only apparent from the deceased's medical history, such as epilepsy, hypoglycaemia and cardiac arrhythmias, should be considered.

(3) What was the cause of death? Although most bodies recovered from water have died from its inhalation, the individual could have fallen into it after collapse and death from natural causes. The possibility of death from the actions of an assailant followed by immersion ("dumping") in water as a means of disposal must always be considered.

(4) Could the deceased's actions before entering the water or once in it have been modified by the influence of alcohol or other drugs? Here, the case for requesting routine toxicological analyses is strong-if only to facilitate interpretation of circumstances surrounding the death.

\section{Death certification}

Once the questions considered above have been answered satisfactorily by the pathologist, formal death certification is required. This may be straightforward (Ia drowning; or Ia vagal inhibition, due to Ib submersion in water; or when death resulted entirely from natural causes). But when drowning is associated with natural diseases or drugs it may be difficult, and the pathologist needs to appreciate the implications of using the standard death certificate format. ${ }^{31}$ If it is thought that death from submersion in water was the direct result of natural disease or intoxication by drugs it should be so certified (Ia drowning, due to Ib intracerebral haemorrhage, due to Ic essential hypertension). But if the pathologist believes that, given all pathological and circumstantial evidence available, death from submersion occurred regardless of any natural disease or intoxication present, then only the mechanism responsible for death should appear on the certificate. ${ }^{2}$ It must be remembered firstly that individuals can die with and not necessarily from diseases and conditions found at post 
mortem examination. Secondly, at present, the death certificate used in the United Kingdom does not allow for the inclusion of conditions which have not caused or contributed significantly to death. ${ }^{31}$

If the pathological findings are negative, ambiguous, or obscured by advanced decomposition certification as "unascertained" (a term understood and accepted by coroners ${ }^{32}$ ) may be honest, accurate and entirely appropriate. ${ }^{31}$ The qualification "appearances here are entirely consistent with drowning" or "appearances here are entirely consistent with death following immersion in water" may be helpful and appreciated by the investigating authorities.

\section{Circumstances: accident, suicide or homicide?}

In practice, almost all deaths after submersion in water are either accidental or suicidal; only a few are homicidal. These questions have been addressed elsewhere ${ }^{1479}$ but are worth considering briefly here. Sometimes the question is more complicated in theory than in practice, as strong collateral evidence may render medical data of secondary importance. ${ }^{9}$

Accidental deaths predominate, and occur under a wide range of circumstances. ${ }^{4}$ In a substantial minority, perhaps $20 \%$ or even more, particularly among the young adult age groups, the victim is under the influence of

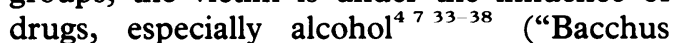
hath drowned more men than Nepture" ${ }^{35}$ ). Here, sudden cooling of skin which is warmer than normal because of vasodilatation may be an important factor in deaths both from drowning and from vagal inhibition. In many of the remainder precipitation by clinically important natural disease may be relevant.

Suicidal deaths are probably commoner than is appreciated or acknowledged, ${ }^{49}$ but returning a verdict of suicide in the absence of confirmatory or good circumstantial evidence is obviously inappropriate and unfair to surviving relatives. It is worth remembering that individuals who commit suicide may first resort to alcohol or other drugs for "courage" and that suicides may have substantial natural diseases. ${ }^{1}$

Homicidal deaths are uncommon ${ }^{40}$ as it requires a considerable physical disparity between the assailant and the victim, or for the victim to be incapacitated by disease, drink, or drugs, or for the victim to be taken by surprise. ${ }^{6}$ Nevertheless, the pathologist must actively consider and positively exclude this possibility in every body recovered from water; only then will missed homicides be minimised, although without evidence of violence, the presumption must be that death was accidental or suicidal. ${ }^{6}$ Therefore, it is essential that all injuries on and within the body are documented and subsequently explained to the complete satisfaction of all parties-pathologists, investigating police officers and Coroner/ procurator fiscal. When any doubts exist, it is wise to engage a specialist forensic pathologist at an early stage.

\section{Deaths in the bath}

These may present particular problems, and always require adequate explanation. ${ }^{4124143}$

Such deaths may, of course, be unrelated to inhalation of bath water-for example from natural causes, overdose of drugs or, occasionally, electrocution. When water inhalation is considered relevant by the pathologist, the death may, as discussed above, be accidental, suicidal, or homicidal. Precipitation into the water by natural disease should always be considered, as should the influence of poisoning, not only by alcohol or drugs, but also by carbon monoxide from faulty water heaters.

With apparently accidental deaths, many pathologists would agree with Cameron that "a normal healthy conscious person does not drown accidentally and that the possibility of such an accident occurring from falling asleep is a convenient, but virtually unsubstantiated, myth." 12

Some authors believe that adult deaths in the bath are most likely to be suicidal ${ }^{42}$; others consider suicide by self immersion to be rare. ${ }^{12}$ In infancy and early childhood, although most deaths are accidental and reflect inadequate adult supervision, deliberate immersion is well documented, and should always be considered and investigated accordingly. ${ }^{44} 45$

1 Knight B. The Coroner's autopsy. A guide to non-criminal autopsies for the general pathologist. Edinburgh: Churchill Livingstone, 1983:251-68.

2 Davis JH. Bodies found in the water. An investigative approach. Am f Forens Med Pathol 1986;7:291-7.

3 Lawler W. The negative coroner's necropsy: a personal approach and consideration of difficulties. 7 Clin Pathol 1990;40:977-80.

4 Giertsen JC. In: Tedeschi CG, Eckert WG, Tedeschi LG, eds. Forensic medicine. Philadelphia: WB Saunders $\mathrm{Co}$ 1977:1317-33.

5 Pullar P. In: Mant AK, ed. Taylor's principles and practice of medical jurisprudence. 13 th ed. Edinburgh: Churchil Livingstone, 1984:292-303.

6 Polson CJ, Gee DJ, Knight B. The essentials of forensic medicine. 4th ed. Oxford: Pergammon Press, 1985: 421-48.

7 Gordon I, Shapiro HA, Berson SD. Forensic medicine. A guide to principles. 3rd edn. Edinburgh: Churchill Living stone, 1988:115-25.

8 Knight B. Forensic pathology. London: Edward Arnold, 1991:360-74.

9 Simpson K. In: Simpson K, ed. Taylor's principles and practice of medical jurisprudence. 12th edn. London: Churchill, 1965:368-83.

10 Keatinge WR, Prys-Roberts C, Cooper KE, Honour AJ, Haight J. Sudden failure of swimming in cold water. $B$ Haight J. Sudden fail

11 Keatinge WR. Hypothermia at sea. Med Sci Law 1984 24:160-2.

12 Cameron JM. In: Camps FE, ed. Gradwohl's legal medicine. 3rd edn. Bristol: John Wright, 1976:349-55.

13 Simpson K. Deaths from vagal inhibition. Lancet 1949; i: $558-60$.

14 Spilsbury B. Some medico-legal aspects of shock. MedicoLegal and Criminological Review 1934;2:1-13.

15 Gardner E. Mechanism of certain forms of sudden death in medico-legal practice. Medico-Legal and Criminological Review 1942;10:120-33.

16 Anonymous. Immersion or drowning? [Editorial] $\mathrm{Br} \mathrm{Med} \mathscr{f}$ 1981;282:1340-1.

17 Donald KW. Drowning. Br Med f 1955;ii:155-60.

18 Fuller RH. Drowning and the postimmersion syndrome. A clinicopathologic study. Military Med 1963;128:22-36.

9 Pearn JH. Secondary drowning in children. Br Med $f$ 1980;281:1103-5.

20 Gordon I. The anatomical signs in drowning. A critical evaluation. Forens Sci 1972;1:389-95.

21 Copeland AR. An assessment of lung weights in drownin cases. The Metro Dade experience from 1978 to 1982 . Am $\mathcal{f}$ Forens Med Pathol 1985;6:301-4.

22 Timperman J. Medico-legal problems in death by drowning. Its diagnosis by the diatom method. $\mathcal{F}$ Forensic Med 1969;16:45-75.

23 Hendey NI. The diagnostic value of diatoms in drowning. Med Sci Law 1973;13:23-34.

24 Peabody AJ. Diatoms and drowning - a review. Med Sci Law 1980;20:254-61. 
25 Calder IM. An evaluation of the diatom test in deaths of professional divers. Med Sci Law 1984;24:41-6.

26 Foged N. Diatoms and drowning-once more Forens Sci Int 1983;21:153-9.

27 Hendey NI. Diatoms and drowning - a review. Med Sci Law 1980;20:289.

28 Gettler AO. A method for the determination of death by drowning. FAMA 1921;77:1650-2.

29 Fisher IL. Chloride determination of heart blood. Its use for the identification of death caused by drowning. $\mathcal{F}$ Forensic Med 1967;14:108-12.

30 Modell JH, Davis JH. Electrolyte changes in human drowning victims. Anesthesiology 1969;30:414-20.

31 Knight B. The Coroner's autopsy. $A$ guide to non-criminal autopsies for the general pathologist. Edinburgh: Churchill autopsies for the general pact
Livingstone, 1983:53-60.

32 Burton JDK, Chambers DR, Gill PS. Coroners' inquiries-a guide to law and practice. Brentford: Kluwer Law Publications, 1985:87.

33 Giertsen JC. Drowning while under the influence of alcohol. Med Sci Law 1970;10:216-19.

34 Plueckhahn VD. The aetiology of 134 deaths due to "drowning" in Geelong during the years 1957 to 1971 . Med f Aust 1972;ii:1183-7.
35 Plueckhahn VD. Alcohol and accidental submersion from watercraft and surrounds. Med Sci Law 1977;17: 246-50.

36 Anonymous. Drinking and drowning. [Editorial.] $\mathrm{Br} \mathrm{Med} \mathcal{f}$ 1979;i:70-1.

37 Cairns FJ, Koelmeyer TD, Smeeton WMI. Deaths from drowning. $N Z$ Med $\mathcal{f} 1984 ; 97: 65-7$.

38 Plueckhahn VD. Alcohol and accidental drowning. A 25 year study. Med F Aust 1984;141:22-5.

39 Copeland AR. Suicide by drowning. Am 7 Forens Med Patho 1987;8:18-22.

40 Copeland AR. Homicidal drowning. Forens Sci Int 1986; 31:247-52.

41 Gardner E. Death in the bathroom. Medico-legal and Criminological Review 1944;12:180-93.

42 Geertinger P, Voigt J. Death in the bath. 7 Forensic Med 1970;17:136-47.

43 Devos C, Timperman J, Piette M. Deaths in the bath. Med Sci Law 1985;25:189-200.

44 Nixon J, Pearn J. Non-accidental immersion in bath water: another aspect of child abuse. Br Med f 1977;i:271-2.

45 Pearn JH, Brown J, Wong R, Bart R. Bathtub drownings: report of seven cases. Pediatrics 1979;64:68-70. 